Revista Brasileira de Agricultura Irrigada v.12, nº.7, p. 3096 - 3107, 2018

ISSN 1982-7679 (On-line)

Fortaleza, CE, INOVAGRI - http://www.inovagri.org.br

DOI: $10.7127 /$ rbai.v12n700981

Protocolo 981.18 - 29/06/2018 Aprovado em 07/02/2019

\title{
AJUSTE DAS EQUAÇÕES DE ESTIMATIVA DA EVAPOTRANSPIRAÇÃO DE REFE- RÊNCIA PARA BENTO GONÇALVES E LAGOA VERMELHA - RS
}

\author{
Jocélia Rosa da Silva ${ }^{1}$, Arno Bernardo Heldwein ${ }^{2}$, Fernando Dill Hinnah ${ }^{3}$, Silvane Isabel Brand ${ }^{4}$, \\ Andressa Janaína Puhl ${ }^{5}$, Mateus Leonardi ${ }^{5}$
}

\begin{abstract}
RESUMO
A escolha de um método para a estimativa da evapotranspiração de referência (ETo) depende fundamentalmente das variáveis meteorológicas disponíveis e da precisão da estimativa. O método recomendado como padrão é o de Penman-Monteith, porém uma desvantagem desse método é a necessidade de um grande número de variáveis meteorológicas e maior dificuldade de sua obtenção. Uma alternativa é o emprego de métodos mais simples com menor número de variáveis meteorológicas. Em contrapartida, esses métodos foram desenvolvidos e/ou calibrados para regiões com características climatológicas diferente das encontradas no estado do Rio Grande do Sul, fazendo com que na comparação entre eles e o método de Penman-Monteith se verifiquem alguns erros, o que torna-se necessário calibrar estes métodos para as condições climáticas do Estado para se obter uma estimativa condizente. Este trabalho teve como objetivo comparar os métodos de estimativa da ETo de BenavidesLopez, Camargo, Hargreaves-Samani, Jensen-Haise, Makkink, Priestley-Taylor, Tanner-Pelton e Turc, com o método de estimativa de Penman-Monteith e proceder ao ajuste dos coeficientes desses métodos para melhorar a estimativa da ETo para Bento Gonçalves e Lagoa Vermelha, municípios do estado do Rio Grande do Sul. Os coeficientes ajustados para Bento Gonçalves e Lagoa Vermelha, RS, permitem obter uma melhor precisão na estimativa da ETo do que com os coeficientes originais possibilitam utilizar os métodos alternativos quando há limitação de disponibilidade de dados para estimativa da ETo pelo método de Penman-Monteith, sem ocorrer significativa perda de precisão.
\end{abstract}

Palavras-chave: Penman-Monteith, ETo, irrigação.

\section{ADJUSTMENT OF REFERENCE EVAPOTRANSPIRATION ESTIMATED EQUATIONS FOR BENTO GOLÇALVES AND LAGOA VERMELHA - RS}

\footnotetext{
${ }^{1}$ Eng. Agrônoma, Mestre em Agronomia, Doutoranda em Agronomia, Universidade Federal de Santa Maria, Santa MariaRS. E-mail: joceliarosa.s@gmail.com

2 Eng. Agrônomo, Doutor em Agronomia, Professor, Universidade Federal de Santa Maria, Santa Maria-RS. E-mail: heldweinab@smail.ufsm.br

${ }^{3}$ Eng. Agrônomo, Doutor em Ciências, Universidade de São Paulo - ESALQ, Piracicaba-SP. E-mail: fhinnah@usp.br

${ }^{4}$ Eng. Agrônoma, Mestre em Engenharia Agrícola, Doutoranda em Engenharia de Sistemas Agrícolas, Universidade de São Paulo - ESALQ, Piracicaba-SP. E-mail: silvanebrand@usp.br

${ }^{5}$ Eng. Agrônoma(o), Mestranda(o) em Agronomia, Universidade Federal de Santa Maria, Santa Maria-RS. E-mail: andressa.puhl@hotmail.com ; mateus-leonardi@hotmail.com

*Autor Correspondente.
} 


\begin{abstract}
The choice of a method for this reference evapotranspiration estimated (ETo) depends primarily on weather variables available and the accuracy of the estimate. The method recommended as standard is that Penman-Monteith, but a disadvantage of this method is the need for a large number of weather variables, and greater difficulty in obtaining it. An alternative is the use of simpler methods with fewer meteorological variables. In contrast these methods have been developed and/or calibrated to regions with different climatic characteristics found in the state of Rio Grande do Sul, making the comparison between them and the Penman-Monteith method to check some errors. This study aimed to compare the ETo estimation models Benavides-Lopez, Camargo, Hargreaves-Samani, Jensen-Haise, Makkink, Priestley-Taylor, Tanner-Pelton and Turc, with the estimation method of Penman-Monteith and proceed to the adjustment of the coefficients of these models to improve the estimation of ETo for Bento Goncalves, Lagoa Vermelha, municipalities in the state of Rio Grande do Sul. The coefficients adjusted for Bento Gonçalves and Lagoa Vermelha, RS, allow to obtain a better precision in the estimation of the ETo than with the original coefficients allow to use the alternative methods when there is limitation of the availability of data to estimate the ETo by the Penman-Monteith method, without significant loss of accuracy occurs.
\end{abstract}

Keywords: Penman-Monteith, ETo, irrigation.

\section{INTRODUÇÃO}

O déficit hídrico é responsável pela redução da produtividade de muitas culturas agrícolas, e a irrigação surge como uma alternativa para manter a produtividade (VIOL et al., 2017; GOMES et al., 2015; PAZ et al., 2000), mesmo em períodos com baixa precipitação pluviométrica. Porém quando mal manejada pode resultar em desperdício de água, e causar um efeito reverso devido a saturação do solo, levando a planta a estresse por excesso hídrico. A perda simultânea de água para a atmosfera devido à evaporação do solo e a transpiração das plantas é chamada de evapotranspiração. Sendo a evapotranspiração das culturas umas das variáveis necessárias para determinar a quantidade real de água a ser suprida ao solo no manejo da irrigação.

A precisão na determinação da quantidade de água a ser irrigada garante que as culturas não sofram com estresse hídrico, resultando em maiores produtividades, melhor utilização dos equipamentos de irrigação e menor consumo de energia elétrica, proporcionando ao produtor rural a redução nos custos de produção (SALES, 2008), logo o aumento dos lucros. Á partir de modelos matemáticos, tendo como parâmetros as variáveis meteorológicas, é possível estimar a evapotranspiração de referência (ETo). O método de estimativa da ETo recomendado pela FAO (Food and Agriculture Organization) para uso operacional é o de Penman-
Monteith (PM) modificado pela FAO no seu manual 56 (ALLEN et al., 2006). Porém, esse método exige uma grande quantidade de variáveis meteorológicas, tornando sua aplicação inviável quando não se detém de todas as variáveis meteorológicas.

Como alternativa para estimativa da ETo torna-se viável o emprego de métodos mais simples, ou seja, que necessitam de um menor número de variáveis meteorológicas. Na literatura científica existem vários métodos, como o de Hargreaves e Samani (1982) e o de Camargo (1971). Porém, muitos métodos de estimativa de ETo foram desenvolvidos e/ou calibrados para regiões com características climatológicas diferentes das encontradas no estado do Rio Grande do Sul. O que para o seu emprego eficiente no estado se faz necessário calibrar os coeficientes para as condições climáticas desejadas. A calibração dos coeficientes dos métodos de estimativa da evapotranspiração de referência para o estado do Rio Grande do Sul é rara, no qual para a região de Bento Gonçalves e Lagoa Vermelha não se encontra nenhum método ajustado.

Este trabalho teve como objetivo comparar os métodos de estimativa da evapotranspiração de referência (ETo) de Benavides-Lopez (BL), Camargo (CM), Hargreaves-Samani (HS), JensenHaise (JH), Makkink (MK), Priestley-Taylor (PT), Tanner-Pelton (TP) e Turc (TU), com o método de estimativa de Penman-Monteith e proceder o ajuste 
dos coeficientes dos métodos para melhorar a estimativa da ETo para Bento Gonçalves e Lagoa Vermelha, municípios do estado do Rio Grande do Sul.

\section{MATERIAIS E MÉTODOS}

Foram utilizados dados meteorológicos diários obtidos através das Estações Meteorológicas Automáticas (EMA) e Convencionais (EMC), pertencente ao Oitavo Distrito de Meteorologia do Instituto Nacional de Meteorologia $\left(8^{\circ} \mathrm{DISME} / \mathrm{IN}-\right.$ MET), dos municípios de Bento Gonçalves e Lagoa Vermelha, localizados no estado do Rio Grande do Sul. A EMA e a EMC de Bento Gonçalves estão localizadas a $29^{\circ} 09^{\prime} 52,5^{\prime \prime} \mathrm{S} 51^{\circ} 32^{\prime} 03,1^{\prime \prime}$ O, a uma altitude de 623 metros do nível do mar e a EMA e a EMC de Lagoa Vermelha estão localizadas a $28^{\circ} 13 ' 20,6^{\prime \prime S}$ e $51^{\circ} 30^{\prime} 46,2^{\prime \prime} \mathrm{O}$, a uma altitude de 834 metros do nível do mar. A escolha desses municípios se deu pela presença de EMA e que apresentaram a EMC. Além de serem municípios com diferentes explorações agrícolas, Bento Gonçalves tem a fruticultura como atividade agrícola predominante, e Lagoa Vermelha a produção de grãos. Dos dados das respectivas EMC's foi utilizado apenas o tempo de brilho solar direto, também denominado de insolação diária (n, horas), para o cálculo do saldo de radiação de ondas longas $\left(\mathrm{L}^{*}\right)$.

Foram utilizados os seguintes dados meteorológicos das EMA's: Radiação solar global incidente $\left(\mathrm{K} \downarrow, \mathrm{MJ} \mathrm{m}^{-2} \mathrm{dia}^{-1}\right)$, somatório dos valores horários diários positivos, com posterior transformação de unidades de KJ para MJ; Temperatura média diária do ar $\left(\mathrm{Tméd},{ }^{\circ} \mathrm{C}\right)$, média dos valores horários diários; Temperatura mínima diária do ar (Tmín, ${ }^{\circ} \mathrm{C}$ ), mínima dos valores horários diários; Temperatura máxima diária do ar $\left(\right.$ Tmáx, $\left.{ }^{\circ} \mathrm{C}\right)$, máxima dos valores horários diários; Umidade relativa diária do ar (UR, \%), média dos valores horários diários; e velocidade do vento $\left(\mathrm{u}_{2}, \mathrm{~m} \mathrm{~s}^{-1}\right)$, média dos valores horários diários. Para determinar o saldo de radiação $\left(\mathrm{Q}^{*}, \mathrm{MJ} \mathrm{m}^{-2} \mathrm{dia}^{-1}\right)$, bem como a radiação solar no topo da atmosfera $\left(\mathrm{Ko} \downarrow\right.$, em MJ m $\left.{ }^{-2} \mathrm{dia}^{-1}\right)$ e o déficit de saturação do vapor do ar (d, $\mathrm{kPa})$ foi adotado o procedimento descrito por Allen et al. (2006), utilizando-se os dados meteorológicos das respectivas EMA's, exceto os valores diários de $n$.

Para a realização deste trabalho foram empregados dados meteorológicos do período de 2007 a 2015, tanto para o município de Bento Gonçalves como para o de Lagoa Vermelha, RS. No qual, para o período de 2007 a 2012 os dados foram utilizados para realizar a calibração dos coeficientes das equações de ETo, enquanto para o período de 2013 e 2015 os dados foram utilizados para a validação dos métodos. Em alguns dias, devido a problemas diversos (problemas de leituras, inconsistência de dados, erro operacional, entre outros) não havendo disponibilidade de todas as medições das variáveis meteorológicas, foi optando pelo descarte dos mesmos, para melhor comparação entre os métodos. Os métodos de estimativa da ETo usados para a realização dos ajustes dos coeficientes foram o de Benavides-Lopez (BL), Camargo (CM), HargreavesSamani (HS), Jensen-Haise (JH), Makkink (MK), Priestley-Taylor (PT), Tanner-Pelton (TP) e Turc (TU), apresentados na Tabela 1. Os métodos diferem quanto ao número de variáveis meteorológicas exigidas para a realização do cálculo e todos eles foram comparados ao método de Penman-Monteith (PM). Para a estimativa da ETo pelo método de PM utilizou-se a equação parametrizada, bem como os demais procedimentos descritos por Allen et al. (2006). A estimativa de ETo para cada método foi realizada diariamente, conforme disponibilidade de dados e período analisado (calibração e validação). Para a calibração dos coeficientes dos métodos avaliados, procedeu-se inicialmente a comparação entre os valores calculados da ETo pelo método de PM com os provenientes da estimativa pelos demais métodos.

Posteriormente, a partir da análise dos desvios, procedeu-se o ajuste local dos respectivos coeficientes, adequando-os às condições dos municípios analisados, utilizando-se o assistente de regressão não linear do programa estatístico SigmaPlot. Definido os novos coeficientes procedeu-se a validação dos ajustes dos coeficientes dos métodos analisados. Para a avaliação do desempenho dos ajustes foram analisados, somente na escala diária, o coeficiente de determinação $\left(R^{2}\right), \quad a$

raiz do quadrado médio do erro (RQME) calculada conforme Janssen e Heuberger (1995), índice de concordância de Willmott (d) e o índice de confiança (c) determinado conforme Camargo e Sentelhas (1997). A acurácia das comparações entre os métodos testados e ajustados com o de PM também foi analisada, usando como parâmetros os termos da equação da reta descrita pela equação 
$y=a x+b$, em que o termo "a" é o coeficiente angular e o termo "b" é o coeficiente linear. O método melhor ajustado é aquele que coin- cide o coeficiente angular igual ou o mais próximo a um e o linear igual ou o mais próximo de zero.

Tabela 1. Métodos de estimativa da evapotranspiração de referência avaliados e suas respectivas equações e coeficientes originais.

\begin{tabular}{|c|c|c|c|c|c|}
\hline \multirow{2}{*}{$\begin{array}{l}\text { Métodos Utili- } \\
\text { zados }\end{array}$} & \multirow{2}{*}{$\begin{array}{l}\text { Referência } \\
\text { Original e/ou } \\
\text { citada }\end{array}$} & \multirow[b]{2}{*}{ Equações de estimativa da ETo* } & \multicolumn{3}{|c|}{ Coeficientes originais } \\
\hline & & & A & $\mathrm{b}$ & $\mathrm{c}$ \\
\hline $\begin{array}{l}\text { Penman-Mon- } \\
\text { teith (PM) }\end{array}$ & $\begin{array}{l}\text { Allen et al. } \\
(2006)\end{array}$ & ETo $=\frac{\Delta Q^{*}}{\left(\Delta+\gamma^{*}\right) L}+\frac{900 \gamma \mathrm{u}_{2} \mathrm{~d}}{\left(\Delta+\gamma^{*}\right)(\text { Tméd }+273)}$ & 一 & - & 一 \\
\hline $\begin{array}{l}\text { Benavides-Lo- } \\
\text { pez (BL) }\end{array}$ & $\begin{array}{l}\text { Benavides e } \\
\text { Lopez (1970) }\end{array}$ & ETo $=$ a $10^{\left[\frac{7,5 \text { Tméd }}{(237,5+\text { Tméd })}\right]}(1-0,01 U R)+b$ Tméd $+\mathrm{c}$ & 1,21 & 0,21 & 2,3 \\
\hline Camargo (CM) & $\begin{array}{l}\text { Camargo } \\
(1971)\end{array}$ & ETo $=0,01\left(\frac{\text { Ko } \downarrow}{2,45}\right)$ Tméd & 0,01 & - & 一 \\
\hline $\begin{array}{l}\text { Hargreaves-Sa- } \\
\text { mani (HS) }\end{array}$ & $\begin{array}{l}\text { Hargreaves e } \\
\text { Samani } \\
(1982)\end{array}$ & ETo $=$ a Ko $\downarrow[($ Tmáx - Tmín)0,5] (Tméd + b) & 0,0023 & 17,8 & \\
\hline Jensen-Haise & $\begin{array}{l}\text { Jensen e } \\
\text { Haise (1963) }\end{array}$ & ETo $=\frac{\mathrm{k} \downarrow}{2,45}(\mathrm{a}$ Tméd $+\mathrm{b})$ & 0,0252 & 0,078 & \\
\hline Makkink (MK) & $\begin{array}{l}\text { Makkink } \\
(1957)\end{array}$ & ETo $=\mathrm{a} \frac{\Delta}{\Delta+\gamma} \frac{\mathrm{K} \downarrow}{2,45}-\mathrm{b}$ & 0,61 & 0,12 & \\
\hline $\begin{array}{l}\text { Priestley-Taylor } \\
\text { (PT) }\end{array}$ & $\begin{array}{l}\text { Priestley \& } \\
\text { Taylor (1972) }\end{array}$ & ETo $=\mathrm{a} \frac{\Delta}{\Delta+\gamma} \frac{\mathrm{Q}^{*}}{2,45}$ & 1,26 & - & - \\
\hline $\begin{array}{l}\text { Tanner-Pelton } \\
\text { (TP) }\end{array}$ & $\begin{array}{l}\text { Tanner \& Pel- } \\
\text { ton }(1960)\end{array}$ & ETo $=a \frac{Q^{*}}{2,45}-b$ & 1,12 & 0,11 & - \\
\hline Turc (TU) & Turc (1955) & ETo $=a\left[\frac{\text { Tmáx }}{(\operatorname{Tmáx}+b)}(c+K \downarrow .23,88)\right]$ & 0,013 & 15 & 50 \\
\hline
\end{tabular}

*ETo: evapotranspiração de referência $\left(\mathrm{mm} \mathrm{dia}^{-1}\right) ; \Delta$ é a tangente à curva de pressão de saturação do vapor d'água $\left(\mathrm{kPa}{ }^{\circ} \mathrm{C}^{-1}\right) ; Q^{*}$ é o saldo de radiação $\left(\mathrm{MJ} \mathrm{m}^{-2} \mathrm{~d}^{-1}\right) ; \gamma^{*}=$ constante psicrométrica ajustada em função da parametrização das resistências aerodinâmica e da cultura $=0,0662(1+0,33 . \mathrm{u} 2) ; \mathrm{L}$ o calor latente de evaporação $\left(2,46 \mathrm{MJ} \mathrm{kg}^{-1}\right) ; \gamma$ a constante psicrométrica $\left(0,0662 \mathrm{kPa}^{\circ} \mathrm{C}^{-1}\right) ; \mathrm{u}_{2}$

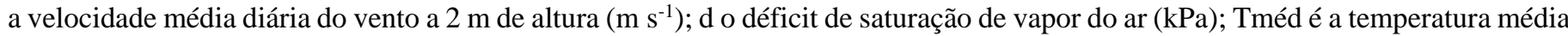
diária $\left({ }^{\circ} \mathrm{C}\right)$; UR a umidade relativa do ar $(\%)$ Ko $\downarrow$ a radiação solar na ausência de atmosfera $\left(\mathrm{MJ} \mathrm{m}^{-2} \mathrm{~d}^{-1}\right)$; Tmáx é a temperatura máxima diária $\left({ }^{\circ} \mathrm{C}\right)$; Tmín é a temperatura mínima diária $\left({ }^{\circ} \mathrm{C}\right)$ e $\mathrm{K} \downarrow$ a radiação solar global incidente $\left(\mathrm{MJ} \mathrm{m}^{-2} \mathrm{~d}^{-1}\right)$.

\section{RESULTADOS E DISCUSSÃO}

Os métodos de estimativa de ETo avaliados e seus respectivos coeficientes originais e ajustados para Bento Gonçalves e Lagoa Vermelha, RS, são apresentadas na Tabela 2. Verifica-se que todos os métodos tiveram ajuste nos seus coeficientes.

Tabela 2. Métodos de estimativa de evapotranspiração de referência avaliados e seus respectivos coeficientes originais e ajustados para Bento Gonçalves (BG) e Lagoa Vermelha (LV), RS.

\begin{tabular}{llllllllll}
\hline $\begin{array}{l}\text { Método } \\
\text { lisado }^{1}\end{array}$ & ana- & \multicolumn{3}{l}{ Coeficientes originais } & \multicolumn{4}{c}{ Coeficientes ajustados $\mathrm{BG}^{2}$} & \multicolumn{3}{c}{ Coeficientes ajustados LV } \\
\hline & a & b & c & a ajust. & b ajust. & c ajust. & a just. & b ajust. & c ajust. \\
\hline BL & 1,21 & 0,21 & 2,3 & 1,4434 & 0,1563 & $-0,8198$ & 1,7558 & 0,1701 & $-0,9354$ \\
CM & 0,01 & - & - & 0,0127 & - & - & 0,0141 & - & - \\
HS & 0,0023 & 17,8 & - & 0,0029 & 8,8972 & - & 0,003 & 9,1727 & - \\
JH & 0,0252 & 0,078 & - & 0,0164 & 0,1599 & - & 0,0166 & 0,1915 & - \\
MK & 0,61 & 0,12 & - & 0,747643 & 0,252006 & - & 0,776112 & 0,0166215 & - \\
PT & 1,26 & - & - & 1,4013 & - & - & 1,4123 & - & - \\
TP & 1,12 & 0,11 & - & 0,9518 & $-0,0618$ & - & 0,9506 & 0,0441 & - \\
TU & 0,013 & 15 & 50 & 0,026 & 57,4641 & 12,0077 & 0,0253 & 52,4613 & 23,5425 \\
\hline
\end{tabular}


$\mathrm{BL}=$ Benavides-Lopes; CM = Camargo; HS= Hargrevis-Samani; JH = Jensen-Haise; MK = Makkink; PT = Priestley-Taylor; TP = Tanner-Pelton; e $\mathrm{TU}=$ Turc.

${ }^{2}$ Coeficientes calibrados (a, b, c) para as condições de Bento Gonçalves (BG), RS.

${ }^{3}$ Coeficientes calibrados $(a, b, c)$ para as condições de Lagoa Vermelha (LV), RS.

Paras os municípios de Bento Gonçalves e Lagoa Vermelha verificou-se que a calibração dos coeficientes pode promover uma considerável melhoria de desempenho dos métodos (Figuras 1, 2, 3 e 4), tendendo a aproximar os coeficientes angulares e lineares das retas de regressão de comparação ao valor de um e zero respectivamente. As melhorias promovidas pelos métodos de ETo com coeficientes ajustados para as duas cidades podem ser
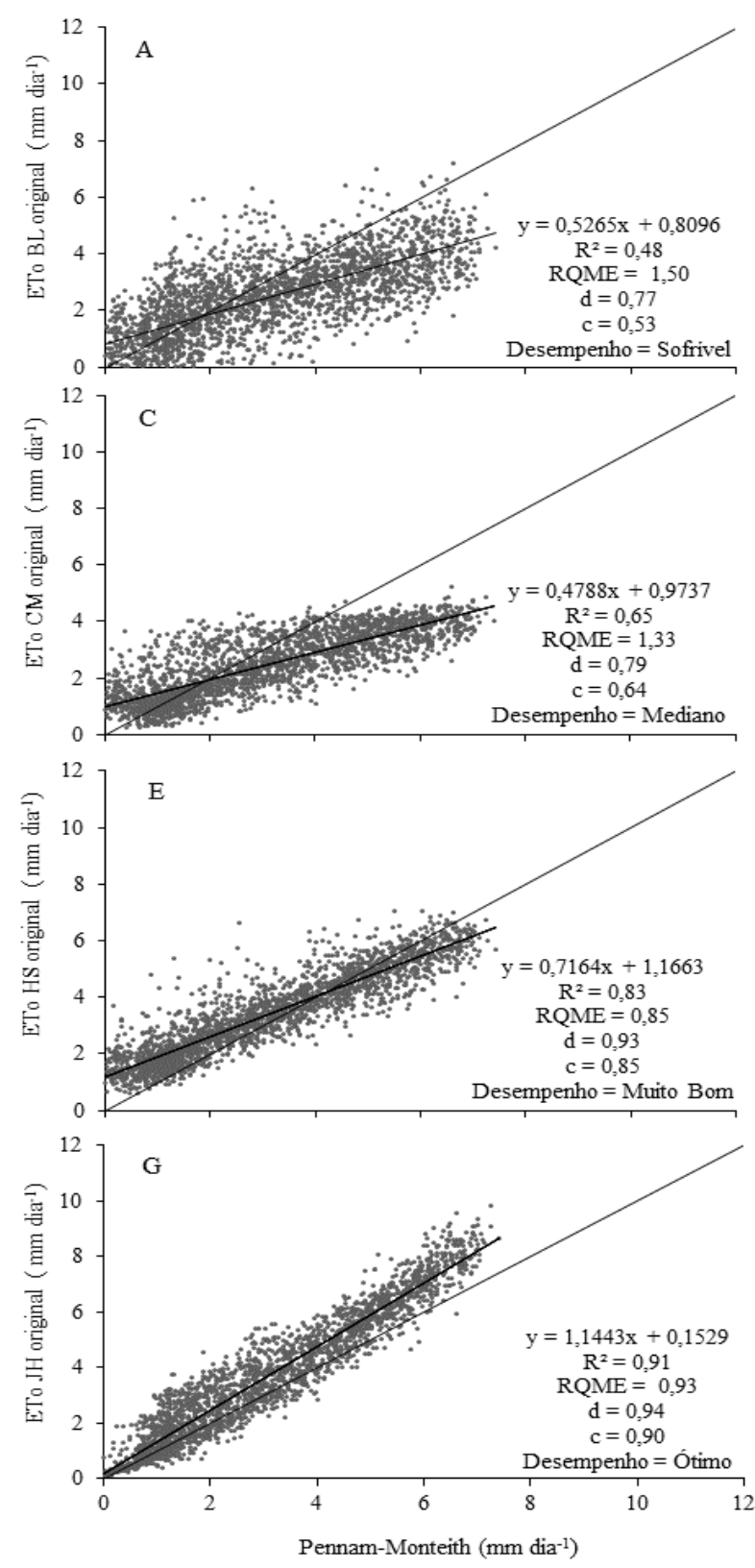

verificadas também ao analisar o coeficiente de determinação $\left(\mathrm{R}^{2}\right)$, que mede a qualidade dos ajustes obtidos quando comparados aos demais métodos de ETo ao PM, o índice de concordância de Willmott (d) e índice de confiança (c), que representam a exatidão e a precisão de um método, e o RMQE que expressa o erro do método, sendo que quanto menor o RQME menor o erro dos métodos (Figuras $1,2,3$ e 4$)$.
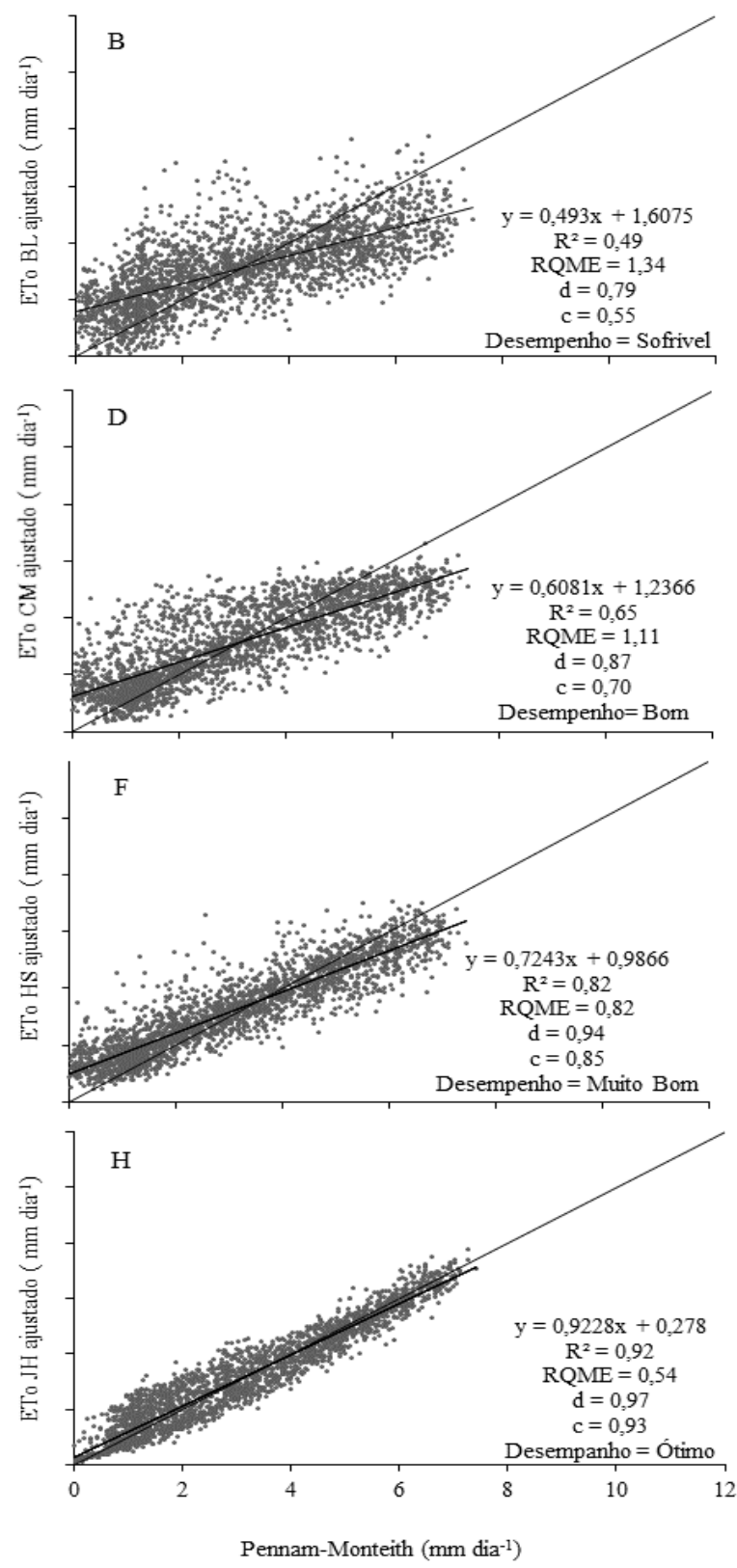

Figura 1. Evapotranspiração de referência diária $\left(\mathrm{ETo}, \mathrm{mm} \mathrm{dia}^{-1}\right)$ estimada pelo método de Penman-Monteith em relação aos métodos de ETo de Benavides-Lopez (BL) com os coeficientes originais ${ }^{1}$ (A), ETo de BL com os coeficientes 
ajustados $^{2}$ (B), ETo de Camargo (CM) com os coeficientes originais ${ }^{1}(\mathrm{C})$, ETo de CM com os coeficientes ajustados ${ }^{2}$ (D), ETo de Hargreaves-Samani (HS) com os coeficientes originais ${ }^{1}$ (E), ETo de HS com os coeficientes ajustados ${ }^{2}$ (F), ETo de Jensen-Haise (JH) com os coeficientes originais ${ }^{1}(\mathrm{G})$ e ETo de $\mathrm{JH}$ com os coeficientes ajustados ${ }^{2}(\mathrm{H})$, com seus respectivos coeficiente de determinação $\left(\mathrm{R}^{2}\right)$, raiz do quadrado médio do erro (RQME, mm dia ${ }^{-1}$ ), índice de Willmott (d), índice de confiança (c) e desempenho do método para o município de Bento Gonçalves, RS.

${ }^{1}$ Período de abrangência dos dados meteorológicos de 2007 a 2012, conforme disponibilidade dos dados. ${ }^{2}$ Período de abrangência dos dados meteorológicos de 2013 a 2015, conforme disponibilidade dos dados.
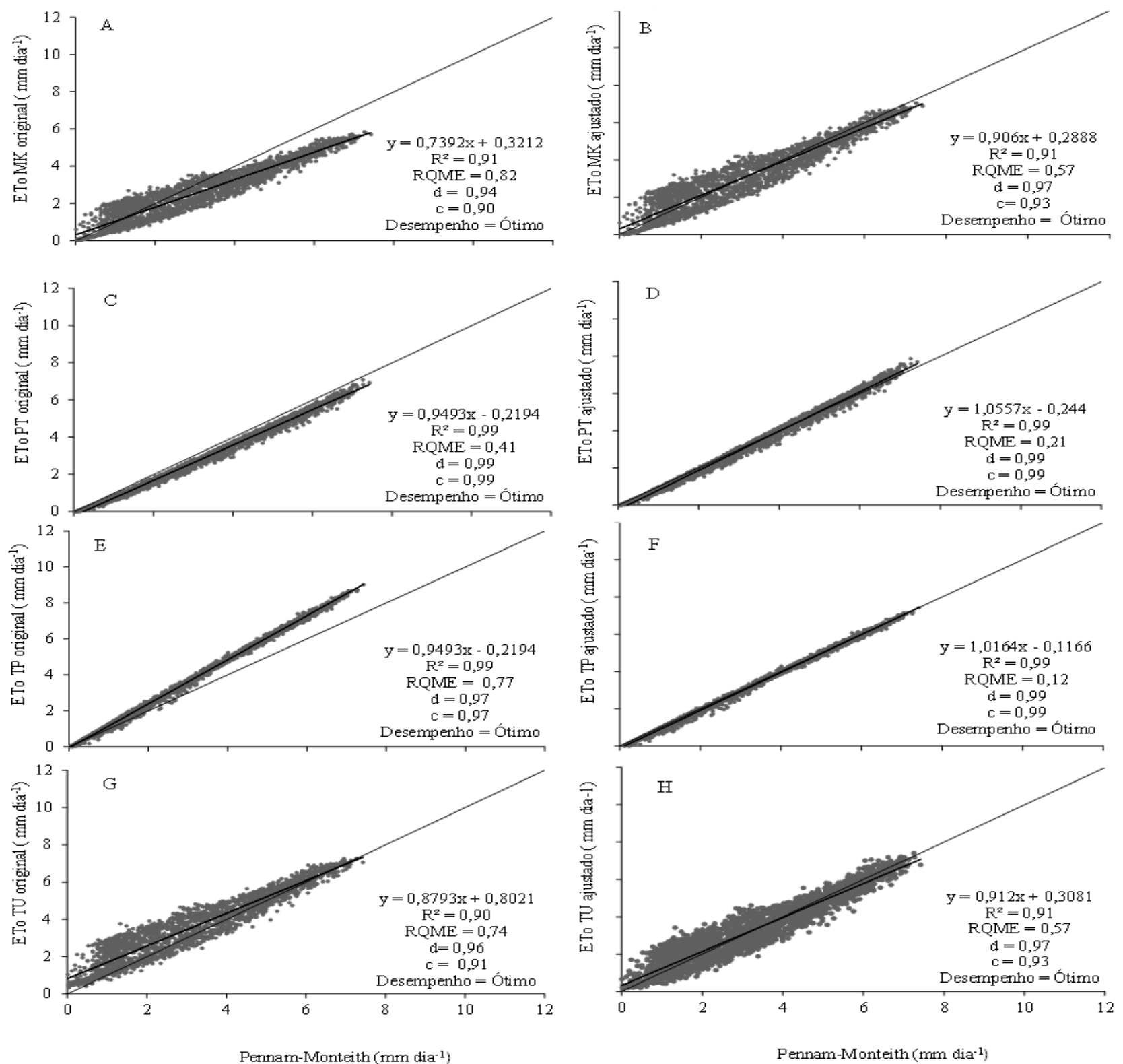

Figura 2 - Evapotranspiração de referência diária $\left(\mathrm{ETo}, \mathrm{mm} \mathrm{dia}^{-1}\right)$ estimada pelo método de Penman-Monteith em relação aos métodos de ETo de Makkink (MK) com os coeficientes originais ${ }^{1}$ (A), ETo de MK com os coeficientes ajustados $^{2}$ (B), ETo de Priestley-Taylor (PT) com os coeficientes originais ${ }^{1}$ (C), ETo PT de com os coeficientes ajustados ${ }^{2}$ (D), ETo de Tanner-Pelton (TP) com os coeficientes originais ${ }^{1}$ (E), ETo de TP com os coeficientes ajustados ${ }^{2}$ (F), ETo de Turc (TU) com os coeficientes originais ${ }^{1}(\mathrm{G})$ e ETo de TU com os coeficientes ajustados ${ }^{2}(\mathrm{H})$, com seus respectivos coeficiente de determinação $\left(\mathrm{R}^{2}\right)$, raiz do quadrado médio do erro (RQME, $\mathrm{mm} \mathrm{dia}^{-1}$ ), índice de Willmott (d), índice de confiança (c) e desempenho do método para o município de Bento Gonçalves, RS.

${ }^{1}$ Período de abrangência dos dados meteorológicos de 2007 a 2012, conforme disponibilidade dos dados. 2Período de abrangência dos dados meteorológicos de 2013 a 2015, conforme disponibilidade dos dados. 

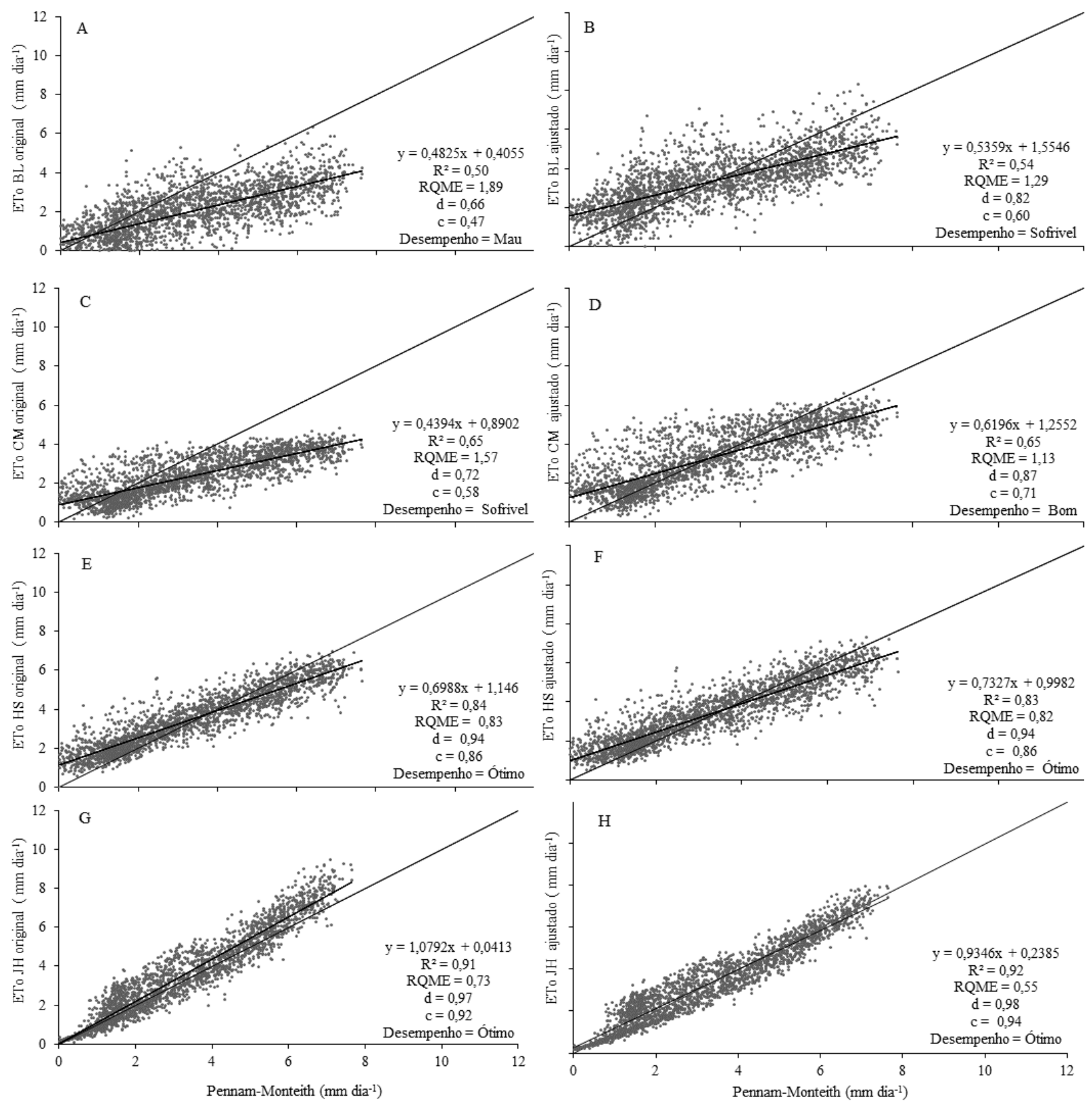

Figura 3. Evapotranspiração de referência diária $\left(\mathrm{ETo}, \mathrm{mm} \mathrm{dia}^{-1}\right)$ estimada pelo método de Penman-Monteith em relação aos métodos de ETo de Benavides-Lopez (BL) com os coeficientes originais ${ }^{1}$ (A), ETo de BL com os coeficientes ajustados $^{2}$ (B), ETo de Camargo (CM) com os coeficientes originais ${ }^{1}(\mathrm{C})$, ETo de CM com os coeficientes ajustados ${ }^{2}$ (D), ETo de Hargreaves-Samani (HS) com os coeficientes originais ${ }^{1}$ (E), ETo de HS com os coeficientes ajustados ${ }^{2}(\mathrm{~F})$, ETo de Jensen-Haise $(\mathrm{JH})$ com os coeficientes originais ${ }^{1}(\mathrm{G})$ e ETo de $\mathrm{JH}$ com os coeficientes ajustados ${ }^{2}(\mathrm{H})$, com seus respectivos coeficiente de determinação $\left(\mathrm{R}^{2}\right)$, raiz do quadrado médio do erro (RQME, $\mathrm{mm} \mathrm{dia}^{-1}$ ), índice de Willmott (d), índice de confiança (c) e desempenho do método para o município de Lagoa Vermelha, RS.

${ }^{1}$ Período de abrangência dos dados meteorológicos de 2007 a 2012, conforme disponibilidade dos dados. 2Período de abrangência dos dados meteorológicos de 2013 a 2015, conforme disponibilidade dos dados. 

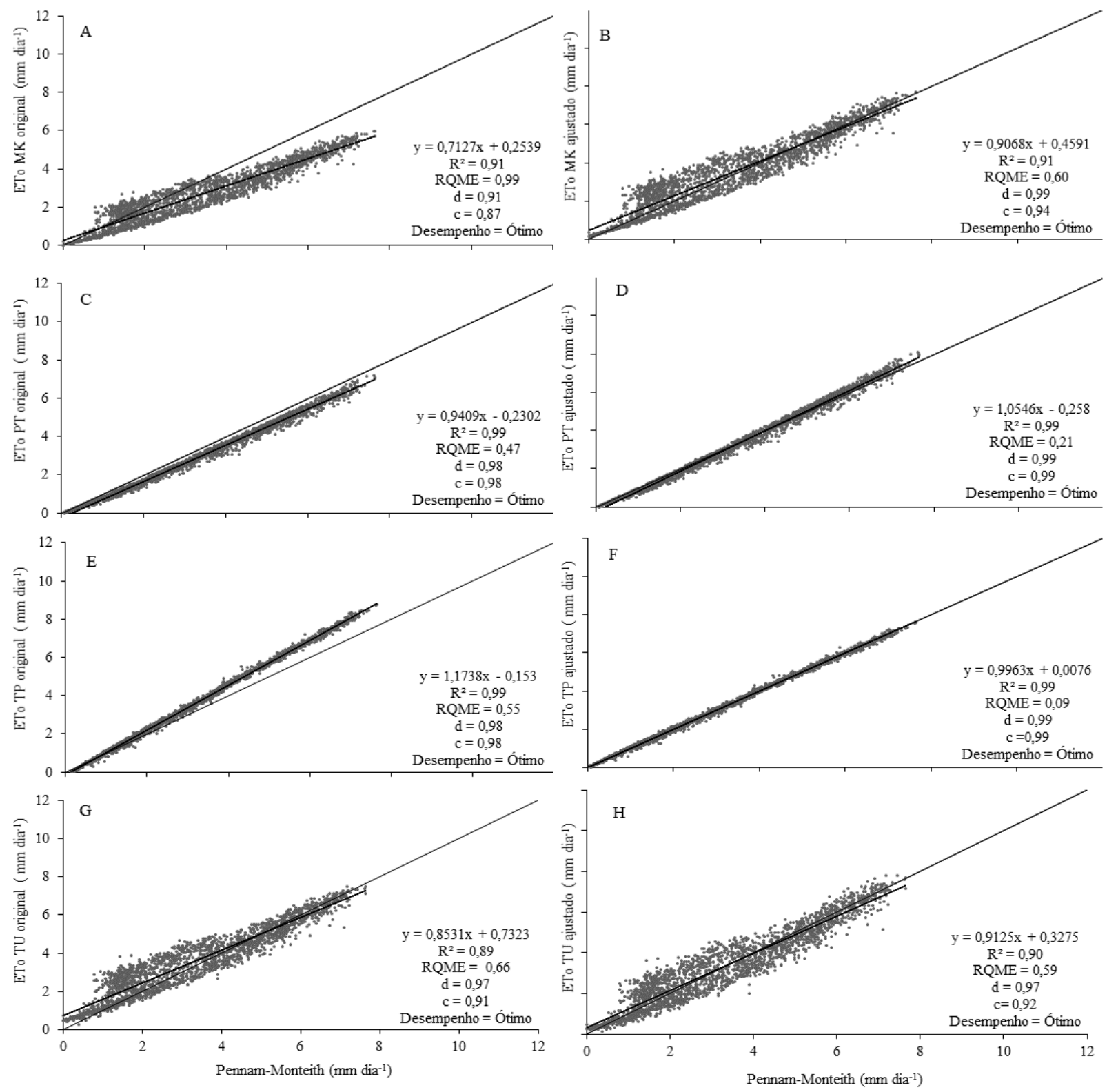

Figura 4. Evapotranspiração de referência diária $\left(\mathrm{ETo}, \mathrm{mm} \mathrm{dia}^{-1}\right)$ estimada pelo método de Penman-Monteith em relação aos métodos de ETo de Makkink (MK) com os coeficientes originais ${ }^{1}$ (A), ETo de MK com os coeficientes ajusta$\operatorname{dos}^{2}(\mathrm{~B})$, ETo de Priestley-Taylor (PT) com os coeficientes originais ${ }^{1}$ (C), ETo de PT com os coeficientes ajustados ${ }^{2}$ (D), ETo de Tanner-Pelton (TP) com os coeficientes originais ${ }^{1}$ (E), ETo de TP com os coeficientes ajustados ${ }^{2}$ (F), ETo de Turc (TU) com os coeficientes originais ${ }^{1}(\mathrm{G})$ e ETo de TU com os coeficientes ajustados ${ }^{2}(\mathrm{H})$, com seus respectivos coeficiente de determinação $\left(\mathrm{R}^{2}\right)$, raiz do quadrado médio do erro (RQME, $\left.\mathrm{mm} \mathrm{dia}{ }^{-1}\right)$, índice de Willmott (d), índice de confiança (c) e desempenho do método para o município de Lagoa Vermelha, RS.

${ }^{1}$ Período de abrangência dos dados meteorológicos de 2007 a 2012, conforme disponibilidade dos dados.

2Período de abrangência dos dados meteorológicos de 2013 a 2015, conforme disponibilidade dos dados.

Para esses municípios todos os ajustes dos coeficientes de estimativa da ETo resultaram na melhoria de pelo menos um dos índices estatísticos resultantes da análise. O método em que se obteve ajuste de maneira precisa e exata, foi o de TP, em que sua linha de tendência praticamente se sobrepõe à linha 1:1. Os métodos de BL para a cidade de Bento Gonçalves (Figuras 1A e 1B) e para Lagoa
Vermelha (Figuras 3A e 3B) e de CM para Bento Gonçalves (Figuras 1C e 1D) e Lagoa Vermelha (Figuras 3C e 3D) apresentaram melhorais quando ajustados os seus coeficientes da equação, com diminuição do RQME, aumento no índice de concordância de Willmott e no índice de confiança, porém em comparação aos demais métodos os seus desempenhos não foram satisfatórios, apresentando 
RQME acima de $1,00 \mathrm{~mm} \mathrm{dia}^{-1}$ mesmo com o ajuste dos coeficientes. Os métodos de ETo de BL e CM tendem a subestimar valores da ETo diária acima de $4 \mathrm{~mm}$, portanto, principalmente em dias com maior demanda atmosférica por vapor d'água. Essa subestimativa pode trazer erros significativos na estimativa de água a ser irrigada para as culturas, ocasionando estresses por déficit hídrico, o que poderá reduzir a produtividade potencial das culturas. Portanto, a estimativa da ETo pelos métodos de BL e CM com coeficientes originais ou ajustados não é recomendado para os municípios de Bento Gonçalves e Lagoa Vermelha quando há a disponibilidade de dados meteorológicos que permitem a utilização de outros métodos.

O método de HS para Bento Gonçalves (Figuras $1 \mathrm{E}$ e 1F) e para Lagoa Vermelha (Figuras 3E e $3 F$ ) tende a subestimar a ETo, o que confere com o encontrado por Brixner et al. (2014), na escala diária, para a região da campanha do estado do Rio Grande do Sul. Apesar de usar no seu método variáveis meteorológicas de obtenção simples, parecidas com os métodos de BL e CM, a estimativa da ETo pelo método de JH é menos imprecisa. Todavia, considerando que mesmo com os ajustes, as estatísticas RQME, $\mathrm{R}^{2}$, o índice de concordância de Willmott e o índice de confiança apresentaram valores similares entre Bento Gonçalves e Lagoa Vermelha, inferindo que os erros de estimativa diária são significativos e o uso desse método deve se restringir a impossibilidade de opção por métodos mais precisos. O método Hargreaves-Samani tende a apresentar melhor desempenho em regiões de clima quente com temperaturas médias do ar altas ao longo do ano, uma vez que o método se baseia nas temperaturas máxima, mínima e média do ar do dia (SOUZA et al., 2014).

$\mathrm{Na}$ ausência de dados de radiação solar ou saldo de radiação, a alternativa recai nos métodos que necessitam somente de dados de temperatura do ar, como o método de Hargreaves-Samani, que foi dentre estes o que apresentou o melhor desempenho, com desempenho considerado muito bom para Bento Gonçalves e ótimo para Lagoa Vermelha. Conceição e Mandelli (2005) afirmam que esse é um dos métodos preferenciais para substituir o método de PM em Bento Gonçalves-RS principalmente quando se dispõe apenas de dados de temperatura do ar. Gonçalves et al. (2009) observaram em estimativas de ETo para o município de Sobral, CE, que o método de HS mostrou ser mais confiável na estimativa da ETo quando comparado aos métodos de JH e CM com os coeficientes originais.

Sem o uso dos coeficientes a, b e c ajustados a equação de $\mathrm{JH}$ tende a superestimar os valores de ETo para ambos os municípios (Figuras $1 \mathrm{G}$ e $2 \mathrm{G}$ ), podendo ocasionar gastos desnecessários em irrigação e excesso hídrico que pode ser evitado com o uso dos coeficientes ajustados. A superestimava da ETo pelo método de $\mathrm{JH}$ se deve ao fato do método ter sido desenvolvido e calibrado em regiões áridas (JENSEN; HAISE, 1963). Com o ajuste dos coeficientes, obteve-se melhora considerável do erro sistemático, diminuindo o desvio dos valores do coeficiente angular e linear, tendendo a um e zero, respectivamente, para ambas as cidades (Figuras 1G, $1 \mathrm{H}, 3 \mathrm{G}$ e $3 \mathrm{H})$. Nessa comparação o $\mathrm{R}^{2}$, o índice de concordância de Willmott e índice de confiança não apresentaram mudança expressiva, porém essa diferença foi mais expressiva no RQME, diminuindo expressivamente. Resultados de superestimava nos valores de ETo calculados pelo método de $\mathrm{JH}$ com os coeficientes originas também foram encontrados por Fanaya Júnior et al. (2012) ao avaliar métodos de estimativa de ETo para o município de Aquidauana, MS. Assim, fica evidenciado que, para as condições brasileiras de clima úmido, os coeficientes da equação de JH precisam ser ajustados.

$\mathrm{O}$ método de MK, que tem em sua equação a variável radiação solar global incidente, apresentou um ótimo desempenho com e sem o ajuste dos coeficientes, porém tendendo a subestimar a ETo para os municípios Bento Gonçalves e de Lagoa Vermelha, quando acompanhada dos coeficientes originais (Figuras 2A e 4A). Com o ajuste dos coeficientes do método (Figuras $2 \mathrm{~B}$ e 4B), obteve-se uma diminuição significativa no valor do RQME, diminuindo assim o erro da estimativa ETo em comparação ao método de PM. O método de PT também apresentou melhor estimativa de ETo quando o coeficiente da equação foi ajustado para Bento Gonçalves (Figuras 2C e 2D) e Lagoa Vermelha (Figuras 4C e 4D) apresentando alta correlação com o método de PM. Sem o ajuste o método tende a subestimar a ETo para ambos os municípios, porém mesmo com o coeficiente original o método apresenta ótimo desempenho.

O coeficiente angular e linear da função linear do teste, além do $\mathrm{R}^{2}$, RQME, índice de concordância de Willmott e o índice de confiança com 
ajuste do coeficiente foram melhorados. Considerando que o método de PT também tem como dados de entrada o saldo de radiação verifica-se que com ajuste dos coeficientes a e b, resultou em excelente desempenho para a estimativa de ETo para os dois municípios, podendo ser uma boa alternativa para a estimativa de ETo na ausência de dados de velocidade do vento.

$\mathrm{O}$ método de TP foi a que apresentou melhor desempenho (menor RQME, maior $\mathrm{R}^{2}$, coeficiente linear e angular da equação de comparação) com os coeficientes ajustados. Com os coeficientes originais, tende a superestimar os valores de ETo de forma crescente para Bento Gonçalves (Figuras 2E e 2F) e Lagoa Vermelha (Figuras 4E e 4F). Fazendo uso dos coeficientes ajustados, o RQME obtido foi de apenas $0,12 \mathrm{~mm} \mathrm{dia}^{-1}$ e de $0,09 \mathrm{~mm} \mathrm{dia}^{-}$ ${ }^{1}$ para Bento Gonçalves e Lagoa Vermelha, respectivamente, inferindo-se que esse método é a melhor alternativa ao método de PM quando não há disponibilidade de dados de velocidade do vento e umidade do ar. Caporusso e Rolim (2015) também consideraram o método de TP o melhor quando o mesmo é comparado ao de PM para Jaboticabal, SP.

Esse resultado conflita com os resultados obtidos por Medeiros (1998), que considera o método de TP com desempenho mediano para o município de Santa Maria, RS, pois para Bento Gonçalves e Lagoa Vermelha também no estado do Rio Grande do Sul esse método obteve desempenho considerado ótimo, tanto com coeficientes ajustados como não ajustados. Da mesma forma Camargo e Sentelhas (1997), também consideram o método de TP com desempenho mediano para estimativas de ETo no estado de São Paulo. É possível que a diferença de desempenho desse método esteja associada à diferença de condição climática entre os locais e, principalmente, em função do uso dos coeficientes originais no método por Medeiros (1998) e Camargo e Sentelhas (1997).

O ajuste dos coeficientes a, b e c (Tabela 2) da equação trouxe melhorias consideráveis ao método de TU para Bento Gonçalves (Figuras $2 \mathrm{G}$ e $2 \mathrm{H}$ ) e Lagoa Vermelha (Figuras 4G e 4H). O teste do método apresentou RQME menor para equação ajustada e maior para a equação com os coeficientes originais.

Cavalcante Junior et al. (2011) em estimativas diárias de ETo para a cidade de Mossoró-RN, classificou os métodos de PT e HS como os melhores métodos e o método de TU como pior, quando comparados ao de PM. Divergindo com os resultados encontrados, em que o método de TU obteve melhor desempenho do que o de HS quando comparados ao método de PM. Isso está relacionado ao clima das regiões analisadas, em que para o RS o clima é classificado como Cfa e o RN como BSw'h'.

Quando os dados meteorológicos disponíveis são de radiação solar global incidente e temperatura média ou máxima diária do ar os métodos alternativos ao de PM são os métodos de JH, MK e TU, que conferem boa aproximação ao método padrão quando ajustados para Bento Gonçalves e Lagoa Vermelha. As estatísticas $\mathrm{R}^{2}$, o índice de concordância de Willmott e o índice de confiança obtidos no teste dos três métodos com coeficientes ajustados foram maiores que 0,90 e o RQME menor que $0,60 \mathrm{~mm} \mathrm{dia}^{-1}$. Porém a maior precisão e exatidão são obtidos pelos métodos de PT e TP, pois ambos levam em consideração o saldo de radiação.

Os resultados encontrados neste trabalho divergem dos resultados obtidos por Conceição e Mandelli (2005) e Medeiros (1998), pois nestes trabalhos a radiação solar global incidente era estimada em função da insolação, que tinha os seus coeficientes de Angtröm-Prescott ajustados com base na radiação solar global medida pelo equipamento actinógrafo tipo Rubitz. Segundo Buriol et al. (2012), o actinógrafo é causador de inconsistência dos registros de radiação solar global, podendo causar erros significativos nas medidas.

As equações de TP, PT, JH, TU, MK e HS, com os coeficientes ajustados para a cidade de Bento Gonçalves e Lagoa Vermelha devem ser utilizadas preferencialmente nessa ordem de classificação como alternativa para estimar a ETo diária nos casos em que a disponibilidade de dados meteorológicos é limitada para utilizar o método de PM. Tais métodos apresentam melhores resultados pois utilizam dados de saldo de radiação ou a radiação solar global incidente.

Os métodos de BL e CM não são indicados para a estimativa de ETo para os municípios de Bento Gonçalves e Lagoa Vermelha, pois geram grandes erros em sua estimativa, que podem trazer problemas no gerenciamento de sistemas operacionais. 


\section{CONCLUSÕES}

$\mathrm{O}$ ajuste dos coeficientes das equações de estimativa da ETo dos métodos de Camargo, Benavides-Lopez, Hargreaves-Samani, Jensen-Haise, Makkink, Priestley-Taylor, Tanner-Pelton e Turc permite obter uma melhor precisão e exatidão na estimativa da ETo do que com os coeficientes originais para os municípios de Bento Gonçalves e Lagoa Vermelha, RS.

Os métodos de Benavides-Lopez e Camargo, apesar de apresentarem melhorias com o ajuste dos coeficientes da equação dos métodos, devem ser evitados para a estimativa de ETo nos municípios de Bento Gonçalves e Lagoa Vermelha, RS.

Os coeficientes ajustados para os municípios de Bento Gonçalves e Lagoa Vermelha, RS, possibilitam utilizar os métodos alternativos de estimativa da ETo, como os de Hargreaves-Samani, Jensen-Haise, Makkkink, Priestley-Taylor, Tanner-Pelton e Turc.

O método de Tanner-Pelton, com coeficientes ajustados para os dois locais, é o mais adequado para estimar ETo quando há limitação de disponibilidade de dados de velocidade do vento e umidade do ar em comparação ao método de PenmanMonteith para os municípios de Bento Gonçalves e Lagoa Vermelha, RS.

\section{REFERÊNCIAS BIBLIOGRÁFICAS}

ALLEN, R.G.; PRUITT, W.O.; WRIGHT, J.L.; HOWELL, T.A.; VENTURA, F.; SNYDER, R.; ITENFISU, D.; STEDUTO, P.; BERENGENA, J.; BASELGA, Y.J.; SMITH, M.; PEREIRA, L.S.; RAES, D.; PERRIER, A.; ALVES, I.; WALTER, I.; ELLIOTT, R. A recommendation on standardized surface resistance for hourly calculation of reference ETo by FAO56 Penman-Monteith method. Agriculture Water Manage, p. 1-22, 2006. https://doi.org/10.1016/j.agwat.2005.03.007

BENAVIDES G.; LOPEZ DIAZ, J. Fórmula para calcular a evapotranspiração potencial adaptada aos trópicos $\left(15^{\circ} \mathrm{N}_{-} 15^{\circ} \mathrm{S}\right)$. Tropical Agronornía, v.20, n.5, p.335-345, 1970.
BRIXNER, G.F; SCHÖFFEL, E.R; TONIETTO, J. Determinação da evapotranspiração por diferentes métodos e sua aplicação no índice de seca na Campanha Gaúcha, Brasil. Revista brasileira de Fruticultura, vol. 36, n.4, p. 780-793, 2014. http://dx.doi.org/10.1590/0100-2945-381/13

BURIOL, G.A.; ESTEFANEL, V.; HELDWEIN, A.B.; PRESTES, S.D.; HORN, J.F.C. Estimativa da radiação solar global a partir dos dados de insolação, para Santa Maria -RS. Ciência Rural, v.42, p. 1563-1567, 2012. http://dx.doi.org/10.1590/ S0103-84782012005000059.

CAMARGO, A. P. Balanço hídrico no estado de São Paulo. 3.ed. Campinas: IAC, 1971. 24 p. Boletim n.116.

CAMARGO, A. P.; SENTELHAS, P.C. Avaliação do desempenho de diferentes métodos de estimativa da evapotranspiração potencial no Estado de São Paulo, Brasil. Revista Brasileira de Agrometeorologia, v. 5, p. 89-97, 1997.

CAPORUSSO, N.B.; ROLIM, G.S. Reference evapotranspiration models using different time scales in the Jaboticabal region of São Paulo, Brazil. Acta Scientiarum Agronomy, v. 37, n. 1, p. 1-9, 2015. http://dx.doi.org/10.4025/a agron.v37i1.18277.

CAVALCANTI JUNIOR, E.G.; ALMEIDA, B.M.; OLIVEIRA, A.D.; SOBRINHO, J.E.; ARAÚJO, E.M.; VIEIRA. R.Y.M. Estimativa da evapotranspiração de referência para a cidade de Mossoró-RN. Revista Brasileira de Agricultura Irrigada, v. 4, n. 2, p. 87-92, 2010. http://dx.doi.org/10.7127/RBAI.V4N200110.

CONCEIÇÃO, M.A.F.; MANDELLI, F. Comparação entre métodos de estimativa da evapotranspiração de referência em Bento Gonçalves, RS. Revista Brasileira de Agrometeorologia, v. 13, n. 2, p. 303-307, 2005.

FANAYA JÚNIOR, E.D.; LOPES, A.S.; OLIVEIRA, G.Q.; JUNG L.H. Métodos empíricos para estimativa da evapotranspiração de referência para 
Aquidauana, MS. Revista Brasileira de Agricultura Irrigada, v. 17, n. 4, p. 418-434, 2012. http://dx.doi.org/10.15809/irriga.2012v17n4p418.

GOMES, E.P; RICKLI, M.E; CECATO, U. VIEIRA, C.V; SAPIA, J.G; SANCHES, A.C. Produtividade de capim Tifton 85 sob irrigação e doses de nitrogênio. Revista Brasileira de Engenharia Agrícola e Ambiental, vol. 19, n. 4, p. 317-323, 2015. http://dx.doi.org/10.1590/1807-1929/agriambi.v19n4p317-323.

GONÇALVES, F.M.; FEITOSA, H.O.; CARVALHO, C.M.; GOMES FILHO, R.R.; JÚNIOR, M.V. Comparação de métodos da estimativa da evapotranspiração de referência para o município de Sobral-CE. Revista Brasileira de Agricultura Irrigada. v. 3, n. 2, p. 71-77, 2009. http://dx.doi.org/10.7127/RBAI.V3N200016.

HARGREAVES, G.H.; SAMANI, Z.A. Estimation of potential evapotranspiration. Journal of Irrigation and Drainage, v. 108, n. 3, p. 225-230, 1982.

JANSSEN, P. H. M.; HEUBERGER, P. S. C. Calibration of process - oriented models. Ecological Modelling, v. 83, n. 1-2, p. 55-56, 1995. https://doi.org/10.1016/0304-3800(95)00084-9.

JENSEN, M.E.; HAISE, H.R. Estimating evapotranspiration from solar radiation. Journal of the Irrigation and Drainage Division, v. 89, p. 15-41, 1963.

MAKKINK, G, F. Ekzamento de la formulo de Penman. Netherlands Journal of Agricultural Science. v. 5, p. 290-305, 1957.

MEDEIROS, S.L.P. Avaliação de métodos de esti - mativa da evapotranspiração de referência para a região mesoclimática de Santa Maria - RS. Revista Brasileira de Agrometeorologia. v. 6, n. 1, p. 105 109. 1998.
PAZ, V. P. S.; TEODORO R. E. F.; MENDONÇA, F. C. Recursos hídricos, agricultura irrigada e meio ambiente. Revista Brasileira de Engenharia Agrícola e Ambiental. v. 4, n. 3, p. 465-473, 2000. https://doi.org/10.1590/S14154366200000030002 5.

PRIESTLEY, C. H. B.; TAYLOR, R. J. On the assessment of surfasse heat flux and evaporation using large-scale parameters. Monthly Weather Review, v. 100, n. 2, p. 81-92, 1972.

SALES, J.C. Caracterização climática e comparação de métodos de estimativa de evapotranspiração de referência para regiões do estado do Ceará. 2008. 194 f. Tese (Doutorado) - Universidade Estadual Paulista, Faculdade de Ciências Agronômicas de Botucatu.

SOUZA, J.M.; PEREIRA, L.R.; RAFAEL, A.M.; SILVA, L.D.; REIS, E.F.; BONOMO, R. Comparison of methods for estimating reference evapotranspiration in two locations of, Espirito Santo. Revista Brasileira de Agricultura Irrigada, v. 8, n. $2, \quad$ p. $114-126, \quad 2014$. http://dx.doi.org/10.7127/rbai.v8n200225.

TANNER, C.B.; PELTON, W.L. Potential evapotranspiration estimates by approximate energy balance method of Penman. Journal of Geophysical Research, v. 65, n. 10, p. 3391- 3413, 1960. https://doi.org/10.1029/JZ065i010p03391.

TURC, L. Estimation of irrigation water requirements, potential evapotranspiration: a simple climatic formula evolved up to date. Annals of Agronomy, v. 12, n. 1, p. 13-49, 1961.

VIOL, A.M; CARVALHO, J.A; LIMA, E.M.C; REZENDE, F.C; MATTOS, R.W. P; RODRIGUES, J.L.M. Déficit hídrico e produção do tomate cultivado em ambiente protegido. Revista Brasileira de Agricultura Irrigada, vol. 11, n. 1, p. $1244 \quad-\quad 1253,2017$. https://doi.org/10.7127/rbai.v11n10058 